\title{
Excimers in the Lowest Rotational Quantum State in Liquid Helium
}

\author{
Luis Guillermo Mendoza-Luna, ${ }^{\dagger}$ Nagham M. K. Shiltagh, ${ }^{\dagger, \S}$ Mark J. Watkins, ${ }^{\dagger}$ \\ Nelly Bonifaci, ${ }^{\ddagger}$ Frédéric Aitken ${ }^{\ddagger}$ and Klaus von Haeften ${ }^{*, \dagger}$
}

$\dagger$ Department of Physics and Astronomy, University of Leicester, University Road, Leicester LE1 7RH, United Kingdom

‡G2ELab-CNRS Equipe MDE 25 Av. des Martyrs BP 166 38042 Grenoble Cedex 9, France

ФPresent address: Departamento de Física, Universidad Autónoma Metropolitana Iztapalapa, Av. San Rafael Atlixco No. 186 Col. Vicentina, C.P. 09340 México D.F., México

$\S$ Department of Physics, College of Science, University of Kerbala, Karbala, Iraq

E-mail: kvh6@le.ac.uk

\begin{abstract}
Evidence for helium excimers $\left(\mathrm{He}_{2}^{*}\right)$ in the lowest allowed rotational quantum state in liquid helium is presented. $\mathrm{He}_{2}^{*}$ was generated by a corona discharge in the gas and normal liquid phases. Fluorescence spectra recorded in the visible region between 3.8 and $5.0 \mathrm{~K}$ and 0.2 and 5.6 bar showed the rotationally resolved $d^{3} \Sigma_{u}^{+} \rightarrow b^{3} \Pi_{g}$ transition of $\mathrm{He}_{2}^{*}$. Analysis of the pressure and temperature dependence of lineshifts and line intensities showed features of solvated $\mathrm{He}_{2}^{*}$ superimposed on its gas phase spectrum and, in the liquid phase only, pressure-induced rotational cooling. These findings suggest that $\mathrm{He}_{2}^{*}$ can be used to investigate bulk helium in different phases at the nanoscale.
\end{abstract}




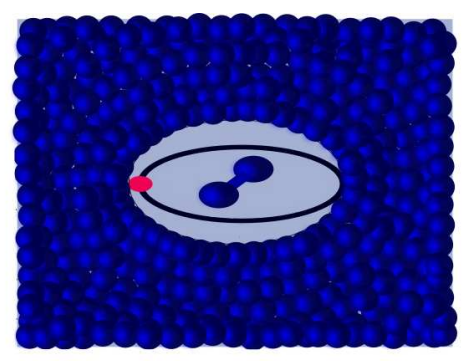

Helium excimer solvated in bulk helium 
Rotational spectroscopy has the power to unravel a considerable amount of detail regarding molecular interactions. Usually, this technique is restricted to studies in the gas phase because rotational resolution cannot generally be achieved in most common liquids. However, a great deal of information has been derived from experiments with helium clusters $^{1-4}$ and droplets in beams, ${ }^{5-7}$ where single foreign molecules can be embedded using the pick-up method. ${ }^{5,8}$ Infrared spectroscopy of these molecules shows sharp lines, indicating free rotation and apparent superfluidity of ${ }^{4} \mathrm{He}$ droplets. ${ }^{9}$ Analysing rotational constants reveals extremely subtle interactions in liquids; for example, an increase of the effective moment of inertia has frequently been observed, which has been attributed to a normal liquid interface around the molecules within the otherwise superfluid ${ }^{4}$ He droplets. ${ }^{10-12}$

An extension of these exceptionally sensitive experiments to bulk helium would benefit from the much wider, and easier, control over pressure, temperature and thermodynamic phase than can otherwise be achieved in helium droplets beams, which have a fixed temperature of $0.4 \mathrm{~K}^{9}\left(0.15 \mathrm{~K}\right.$ in mixed ${ }^{3} \mathrm{He}^{4} \mathrm{He}$ droplets $\left.{ }^{12}\right)$. However, embedding foreign molecules is not trivial because they condense at the temperature of liquid helium. Interestingly, electronically excited condensed helium itself exhibits discrete molecules in the form of helium excimers $\left(\mathrm{He}_{2}^{*}\right)$ - a discovery that has attracted considerable attention. ${ }^{13-23}$ While they are popular as probes for the bulk phases of helium, ${ }^{24-29}$ for example by imaging, ${ }^{30}$ a number of peculiarities are seen that are in stark contrast to the results of the helium droplet work; where for instance, the intensity distribution of rotational lines reflects the temperature of the droplet environment. In contrast, both the fluorescent ${ }^{14,31}$ and transient absorption spectra $^{17}$ of $\mathrm{He}_{2}^{*}$ in liquid helium show the population of high rotational quantum levels corresponding to temperatures of several hundred Kelvin. The work on helium droplets also suggests the existence of equilibrium states, i.e., solvated excimers, in bulk helium, but experimental evidence has not been reported yet to support this supposition.

To advance the understanding of solvation in liquids, we have recorded fluorescence spectra of $\mathrm{He}_{2}^{*}$ solute over a wide range of hydrostatic pressures between 0.2 and 5.6 bar, and at 
temperatures between 3.8 and $5.0 \mathrm{~K}$, covering the gas and normal liquid phases of helium. A corona discharge was chosen for electronic excitation because it shows stable operation over a very wide range of pressures. At low pressures, spectra showed sharp, discrete rotational lines; upon increasing the pressure, the lines broadened and ultimately evolved into broad, poorly resolved features. Furthermore, the intensity distribution shifted towards the lowest rotational state. As this was only seen in the liquid phase, and with a different lineshift than the rest of the spectrum, it was clear that the $\mathrm{He}_{2}^{*}$ spectrum is a superposition of features from gas phase and solvated excimers. This means that not only do solvated excimers exist, they can also be identified via their spectroscopic fingerprint.

Fluorescence spectra recorded at 3.8, 4.0, 4.5 and $5.0 \mathrm{~K}$ showing the $d^{3} \Sigma_{u}^{+} \rightarrow b^{3} \Pi_{g}$ transition of $\mathrm{He}_{2}^{*}$ in the $\mathrm{n}=3$ Rydberg state ${ }^{32}$ are shown in Figure 1 for various different hydrostatic pressures, covering both the gas and the liquid phases of helium. A glow discharge spectrum recorded in the gas cell at very low pressure is shown as a reference for the linepositions under vacuum conditions ${ }^{32}$ and to demonstrate the resolution of the spectrometer. The spectrum shows typical features with P, Q and R rotational lines defined by the selection rules $\Delta N=-1,0$ and 1 , respectively. The quantum number, $N$, denotes the lower rotational state of the transition and starts with the electronic angular momentum quantum number, which is one for the $b^{3} \Pi_{g}$ state. $N$ refers to Hund's coupling case (b), where $\mathbf{N}$ is total angular momentum, J, minus the electron spin, S. P and R lines are resolved; the Q lines lie almost on top of each other and are thus too closely spaced to be fully resolved by our spectrometer. All lines are split into groups of six energetically close transitions due to spinspin and spin-orbit interactions of the triplet levels in Hund's case (b). ${ }^{33}$ As before, however, these splittings cannot be observed at our resolution.

At low cell pressures, sharp, discrete lines are observed, similar to the spectra reported by Hill ${ }^{17}$ and Li. ${ }^{31}$ With increasing pressure, the lines shift in frequency, broaden and change their shape. By comparison with the glow discharge spectrum, it can be clearly seen that at lower pressures the $\mathrm{P}(2)$ line and Q-branch quickly gain intensity compared to the other 


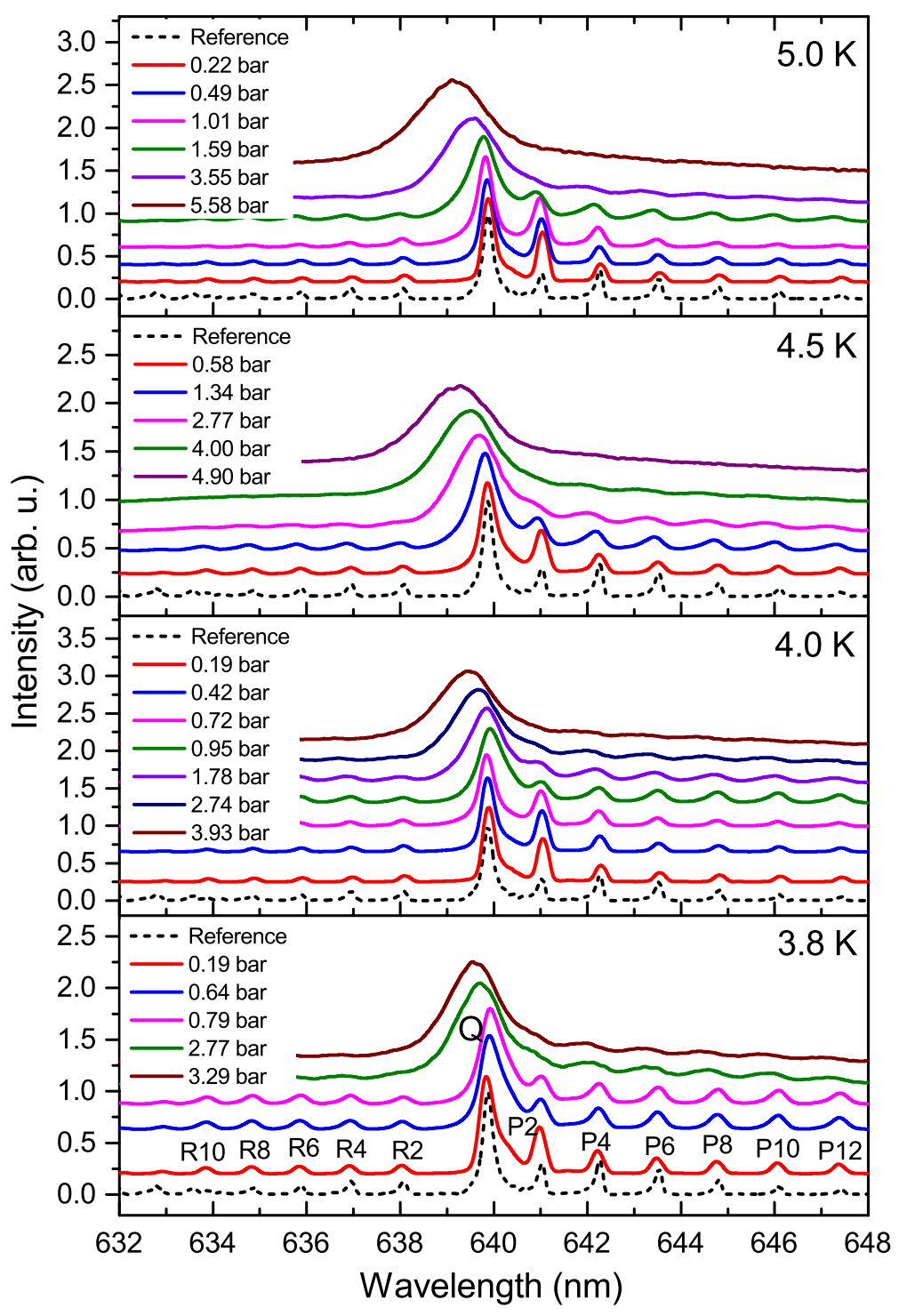

Figure 1: Selection of spectra of $\mathrm{He}_{2}^{*}$ in helium as a function of pressure for four isotherms (the measured temperature deviated by no more than $0.1 \mathrm{~K}$ from the isotherm). The lines of a glow discharge reference spectrum are labelled $\mathrm{R}, \mathrm{Q}, \mathrm{P}$ as a function of quantum number $N$ (Hund's case (b)). With increasing pressure, the lines broaden and shift. Note also that with increasing pressure the $\mathrm{Q}$ and $\mathrm{P}(2)$ lines gain more intensity compared to other lines, and ultimately merge. The intensities and offset have been scaled for better visualisation. 
$\mathrm{P}$ lines. Additionally, the $\mathrm{P}(2)$ line merges at a certain, distinct pressure - for each given isotherm - with the Q-branch. At $3.8 \mathrm{~K}$, this occurs between 0.2 and 0.6 bar, at $4.0 \mathrm{~K}$ between 0.7 and $0.9 \mathrm{bar}$, at $4.5 \mathrm{~K}$ between 1.3 and $2.8 \mathrm{bar}$, and at $5.0 \mathrm{~K}$ between 1.6 and 3.6 bar. Rotational resolution is no longer apparent when pressures increase much beyond the saturated vapour pressure (SVP, which increases from 0.6 bar at $3.8 \mathrm{~K}$ to 1.9 bar at $5.0 \mathrm{~K}$ ) of pure helium, first for $\mathrm{R}$ lines and then for $\mathrm{P}$ lines. At higher pressures, the spectrum is similar to that reported by Dennis et al. ${ }^{14}$ Most of the intensity is concentrated around the band in the centre. We assign these features to the envelope of the $\mathrm{Q}(1)$ and $\mathrm{P}(2)$ transitions. The fact that these two transitions are not resolved is attributed to the normal liquid environment, which was found to hinder rotation and produce a collapsed spectrum for OCS. ${ }^{12,34}$

To analyse the changes in line intensity and line position, the lines were individually fitted using Lorentzian functions. Over a large range of pressures the lines remain symmetrical, hence fitting to a Lorentzian lineshape represents a reasonabl means by which to assess and quantify line intensities and lineshifts. For solvated, and hence cold, $\mathrm{He}_{2}^{*}$, only the lowest allowed quantum state, $N=1$, will be populated because the next highest allowed level, $N=3$, is $75 \mathrm{~cm}^{-1}$ higher in energy. Only odd $N$ rotational states are allowed in the $d^{3} \Sigma_{u}^{+}$ state due to exchange symmetry. For the $d^{3} \Sigma_{u}^{+}$state of $\mathrm{He}_{2}^{*}$, the only transitions emerging from the $N=1$ state are the $\mathrm{Q}(1)$ and $\mathrm{P}(2)$ lines. The intensity ratio between $\mathrm{Q}(\mathrm{N})$ and $\mathrm{P}(\mathrm{N})$ lines $\left(N_{P}>2\right)$ therefore qualitatively reflects the degree of rotational cooling. Figure 2 shows the ratio of the line intensity of the Q-lines and the averaged line intensity of the P-lines. The intensity of the P (2) line has been excluded from the average because it changes line shape and merges with the Q-branch. For all four isotherms shown in Figure 2, the $\mathrm{Q} / \mathrm{P}$ intensity ratio is roughly constant in the gas phase, but increases linearly in the liquid phase of helium with pressure. Presumably, the rotational energy is transferred into collective modes of the solvation shell and liquid environment. ${ }^{35,36}$ In superfluid helium droplets, this type of energy transfer was found to cause rotational line broadening. ${ }^{37,38} \mathrm{~A}$ 


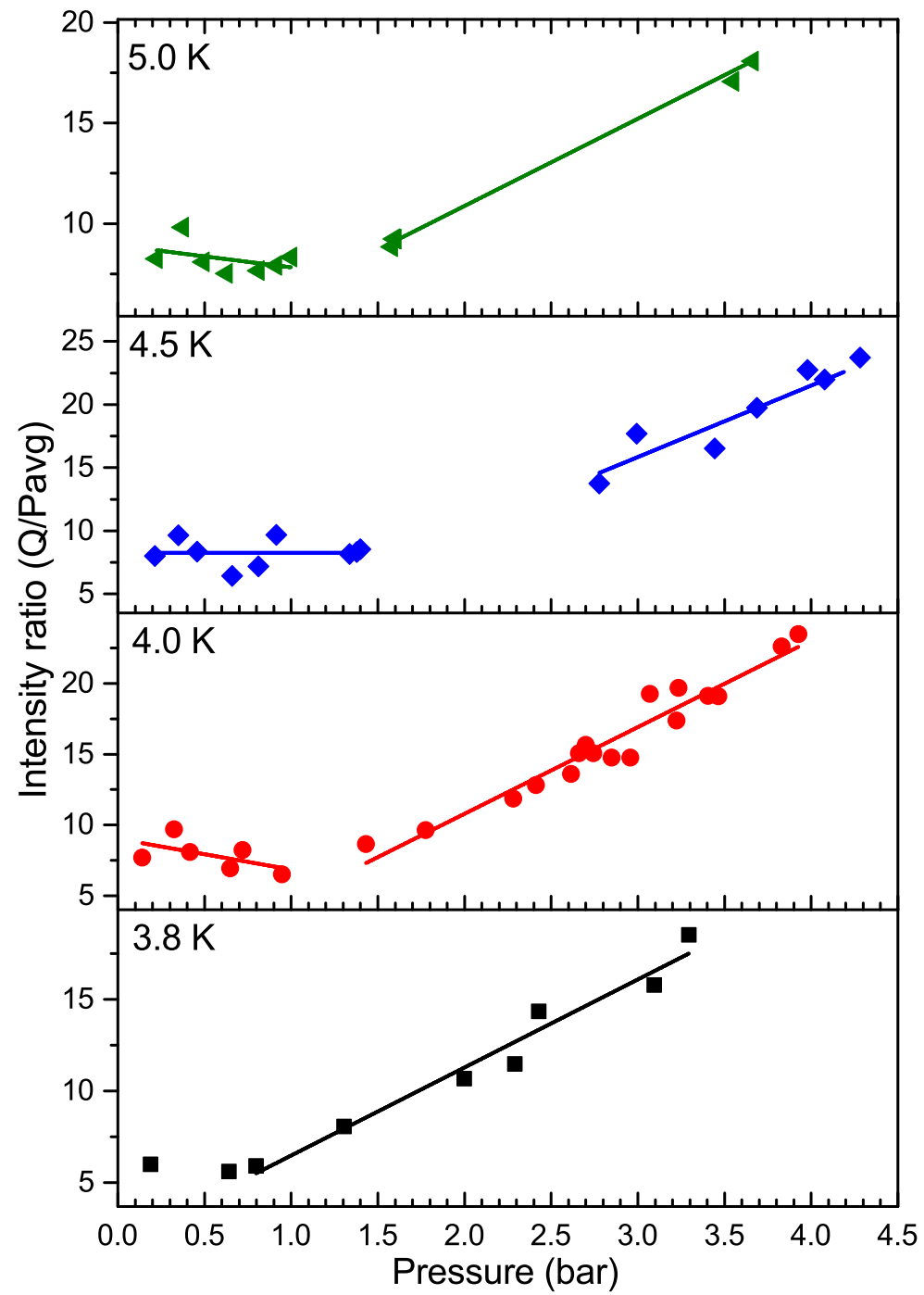

Figure 2: Pressure dependence of the intensity ratio of the Q-branch and averaged P-lines (excluding $\mathrm{P}(2)$, see text) for $3.8,4.0,4.5$ and $5.0 \mathrm{~K}$. The ratio is roughly constant in the gas phase, but increases linearly with pressure in the liquid phase of helium. 
simple explanation of the linear dependence of the $\mathrm{Q} / \mathrm{P}$ intensity ratio on pressure is that molecules coexisting in the gas phase are rotationally cooled by collisions with the liquid. Additionally, the use of electronically excited helium excimers as molecular probes means that our ability to observe rotational cooling is limited by the excimer's radiative lifetime. An upper limit to the fluorescence lifetime has been found as $\tau \sim 10 \mathrm{~ns} .{ }^{39}$ The fact that solvated excimers may cool from several hundred Kelvin to $4 \mathrm{~K}$ within this timeframe would give a lower limit to the rotational cooling rate of $\sim 10^{-10}-10^{-11} \mathrm{Ks}^{-1}$.

Figure 3 shows the Q- and P-lineshifts, $\Delta \lambda(\mathrm{p})$, as a function of pressure. The lineshifts of all sharp P-lines are similar, but dissimilar to the Q-lines. The lineshifts can be grouped into different pressure regions: at pressures below the SVP of liquid helium, the Q-lines show small, and only slightly increasing shifts when the pressure is increased. When the pressure is higher than the SVP of liquid helium, and when subsequently further increased, the Q-lines exhibit a stronger lineshift, which we attribute to stronger perturbation. Interestingly, at 4.0 K, a small but abrupt red shift is seen close to the SVP of liquid helium. The same red shift appears to be present at $3.8 \mathrm{~K}$, but more data points would be needed to substantiate this supposition. The observed abrupt changes indicate a change in the structure of the perturbing environment; they correlate with the changes of the intensity ratio of the $\mathrm{Q}$ and $\mathrm{P}$ lines and are, therefore, attributed to the formation of a dense solvation shell. Lineshift coefficients, $\frac{\partial \Delta \lambda}{\partial p}$ and $\Delta \lambda_{0}$, were obtained for the gas and solvated phases separately by fitting the function $\Delta \lambda(\mathrm{p})=\frac{\partial \Delta \lambda}{\partial p} p+\Delta \lambda_{0}$ to the data, as shown in table 1, with the exception of the $3.8 \mathrm{~K}$ isotherm, where at pressures before the red shift occurs there is only one data point available.

Table 1 shows similar lineshift coefficients for $\mathrm{Q}$ and $\mathrm{P}_{\text {avg }}$ transitions in the gas phase, as expected. In the liquid phase, however, the lineshift coefficients of the $\mathrm{Q}$ and $\mathrm{P}_{\text {avg }}$ transitions differ and diverge, having different slopes. This indicates that the $\mathrm{P}$ and $\mathrm{Q}$ features must originate from different species, i.e., species in different environments, because in molecular spectra the positions of the $\mathrm{P}$ and $\mathrm{Q}$ lines are mutually dependent, as defined by the ap- 


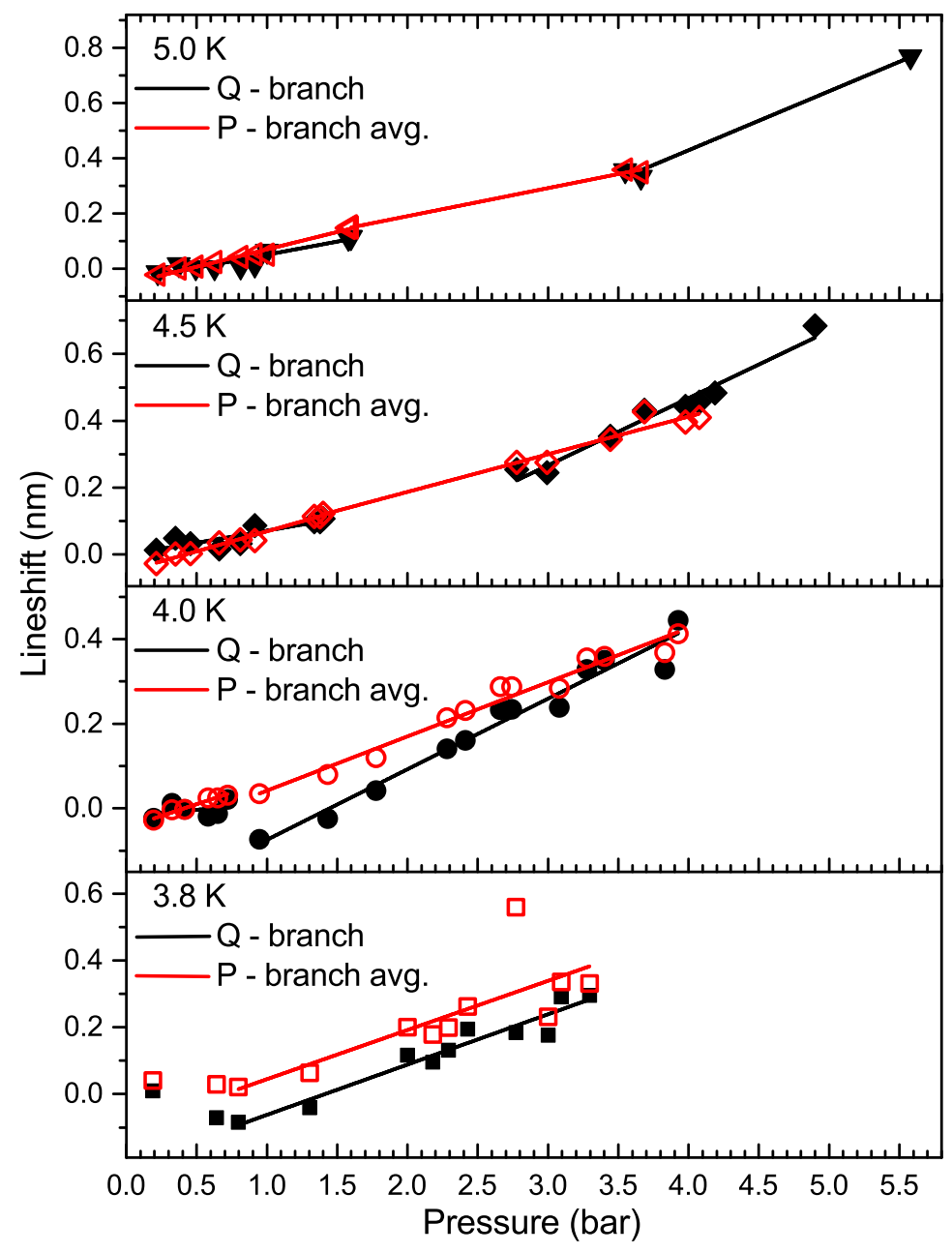

Figure 3: Lineshifts as obtained through fitting with Lorentzians. Filled symbols represent 'Q-transitions', whilst hollow symbols represent P-transitions averaged over all $N$ except $N=2$ (see text). The lineshifts can be grouped according to the gas and liquid phases of the helium. The $\mathrm{P}_{\text {avg }}$ lineshift coefficients (slope of straight lines) do not change between the gas and liquid phase, but the Q lineshift coefficients are consistently larger in the liquid phase, indicating stronger perturbation. 
Table 1: Lineshift coefficients for $\mathrm{Q}$ and averaged $\mathrm{P}(N>2)$ lines in the gas phase and liquid regions. Coefficients could not be determined at $3.8 \mathrm{~K}, \mathrm{Q}_{\text {gas }}, \mathrm{P}_{\text {gas }}$ (too few data) and $5.0 \mathrm{~K}$, $\mathrm{P}_{\text {liq }}$ (unresolved lines).

$\begin{array}{lcccc} & & & \begin{array}{c}\text { slope, } \frac{\partial \Delta \lambda}{\partial p} \\ \text { T }(\mathrm{K})\end{array} & \text { intercept, } \Delta \lambda_{0} \\ 4.0 & & & {[\mathrm{~nm} / \mathrm{bar}]} & {[\mathrm{nm}]} \\ 4.0 & \text { (P) } & \text { gas } & 0.029 \pm 0.040 & -0.018 \pm 0.021 \\ 4.5 & \text { gas } & 0.110 \pm 0.011 & -0.045 \pm 0.006 \\ 4.5 & \text { (P) } & \text { gas } & 0.073 \pm 0.015 & 0.002 \pm 0.014 \\ 5.0 & \text { gas } & 0.119 \pm 0.007 & -0.050 \pm 0.006 \\ 5.0 & \text { (P) } & \text { gas } & 0.095 \pm 0.011 & -0.044 \pm 0.012 \\ 3.8 & \text { gas } & 0.126 \pm 0.006 & -0.056 \pm 0.006 \\ 3.8 & \text { (P) } & \text { liq. } & 0.151 \pm 0.015 & -0.213 \pm 0.035 \\ 4.0 & \text { liq. } & 0.147 \pm 0.043 & -0.103 \pm 0.104 \\ 4.0 & \text { (P) } & \text { liq. } & 0.167 \pm 0.010 & -0.241 \pm 0.029 \\ 4.5 & \text { liq. } & 0.128 \pm 0.007 & -0.086 \pm 0.020 \\ 4.5 & \text { (P) } & \text { liq. } & 0.201 \pm 0.016 & -0.337 \pm 0.061 \\ 5.0 & \text { liq. } & 0.113 \pm 0.011 & -0.038 \pm 0.038 \\ & \text { (Q) } & \text { liq. } & 0.214 \pm 0.021 & -0.426 \pm 0.091\end{array}$

propriate rotational constants (and hence, structure) and, therefore, cannot diverge. Our observations can be readily explained by a superposition of spectra from excimers residing (i) in a solvated state, and (ii) in the gas phase. Both species contribute, with different spectral weights, to the $\mathrm{Q}$ and $\mathrm{P}$ lines of the spectrum. These different contributions are also apparent in the significantly larger intensities of the $\mathrm{Q}$ and $\mathrm{P}(2)$ features compared to the $\mathrm{P}$ and $\mathrm{R}$ lines of higher $N$ in figure 1 .

The co-existence of $\mathrm{He}_{2}^{*}$ in the gas phase under conditions where the helium should be liquid is not unreasonable. Similar to the electron beams used in the past, corona discharge excitation in a point-plane geometry concentrates excitation energy within a small volume, which may lead to local heating and boiling in the vicinity of the tip electrode.

The establishment of the spectral fingerprint of solvated helium excimers in liquid helium and the associated pressure- and temperature-dependent lineshift is encouraging for use of molecular probes in the investigation of superfluid helium, for which a number of techniques for the injection of molecules are currently being pursued. ${ }^{40-43}$ In a broader context, such experiments may lead to a better understanding of molecular solvation in related systems ${ }^{44-46}$ 
in particular, and fluids in general.

In conclusion, we have investigated the spectra of $\mathrm{He}_{2}^{*}$ in gaseous and normal liquid helium as a function of pressure and temperature. At low pressure, rotationally resolved lines were observed. The lines shifted in energy and broadened until they completely vanished as pressure was increased. Analysis of the line intensities and lineshifts shows evidence for the presence of cold excimers in their lowest allowed $(N=1)$ quantum state. Their spectra are superimposed with those of rotationally hot excimers in the gas phase, even under conditions where the helium is liquid. The cold excimer spectra display distinct, pressure- and temperature-dependent lineshifts with respect to the gas phase spectra, reflecting different degrees of perturbation and the existence of a solvation shell whose structure depends on the thermodynamic state. An increase in the pressure of the liquid phase of helium was found to increase the cooling rate, which was attributed to energy transfer to collective modes. Our findings show that excimers exist in thermal equilibrium with helium, having a specific pressure- and temperature-dependent fingerprint. The exploration of helium in different phases at the nanoscale with the great precision inherent to rotational spectroscopy should therefore be possible. This is promising for the investigation of molecular superfluidity.

\section{Experimental Method}

The experiment was performed using an entirely new set up. The apparatus was conceptually similar to that described by Li et al., ${ }^{31}$ but employed a closed-cycle Oxford Instruments Heliox AC-V He3 cryostat. A micro-discharge cell, with an internal volume of $4 \mathrm{ml}$ and constructed from oxygen-free copper (OFHC), was attached to the ${ }^{3}$ He stage, but it was subsequently found that sufficient cooling power could only be provided after bridging the second stage with copper strips. With these bridges, the cell could reach a minimum temperature of $3.2 \mathrm{~K}$, as measured by a calibrated Cernox resistor within $\pm 3 \mathrm{mK}{ }^{1}$. The cell

\footnotetext{
${ }^{1}$ On the SVP, the temperature of the helium inside the cell deviated by no more than $0.1 \mathrm{~K}$ on the sensor readout
} 
was equipped with electrodes in a point-plane configuration - the $250 \mathrm{~nm}$-radius tip having been etched from a tungsten wire - with an electrode gap of 3 to $4 \mathrm{~mm}$. After purging and evacuating the lines with a scroll pump, up to 100 bar of N6.0 research grade purity helium was introduced via stainless steel pipes. The pressure was capacitively measured. A discharge was ignited using a Spellman high voltage power supply using negative voltages between 3 and $10 \mathrm{kV}$ connected to the tip electrode, and currents between 0.1 and 10 $\mu \mathrm{A}$ depending on the thermodynamic phase of the helium. Fluorescence emitted from the discharge region was collected by an achromatic lens, which also served as a high pressure window to the cell in order to maximise detection efficiency. The fluorescence was collimated by two further lenses and then guided, via two adjustable, metal-coated mirrors and an $\mathrm{f}=150 \mathrm{~mm}$ achromatic lens, onto the entrance slit of an Andor Technology Shamrock SR303i Czerny Turner spectrometer equipped with a Peltier-cooled $\left(-65^{\circ} \mathrm{C}\right) \mathrm{CCD}$ camera (Andor iDus DV420, CCD-12855). A $1200 \mathrm{~mm}^{-1}$ grating blazed at $500 \mathrm{~nm}$ was employed, centred around $640 \mathrm{~nm}$, and spectra were recorded at a resolution of $0.2 \mathrm{~nm}$. Further details can be found in. ${ }^{47}$

\section{Acknowledgement}

$\mathrm{KvH}$ and FA acknowledge funding by the British Council (Alliance Programme). KvH is grateful for funding by The Leverhulme Trust (Res. Grant F00212AH), the Royal Society (Int. Exchange Grant IE130173), COST action CM 1405 (Molim) and the Université Joseph Fourier for a Visiting Professorship. LGML and NS acknowledge financial support from the Mexican Consejo Nacional de Ciencia y Tecnología (CONACYT) Scholarship No. 310668, ID 215334 and from the Iraqi Ministry of Higher Education and Scientific Research (MoHESR) Scholarship No. 3312/2014 - 1252, respectively. We are indebted to the Space Research Centre at the University of Leicester who kindly lent us the Oxford Instr. closed cycle cryostat (M. Sims, D. Ross, J. Williams, J. Lapington, R. Limpenny) and, finally, numerous 
colleagues for discussions and A. M. Ellis for proof reading the manuscript.

\section{References}

(1) Tang, J.; Xu, Y.; McKellar, A. R. W.; Jäger, W. Quantum Solvation of Carbonyl Sulfide with Helium Atoms. Science 2002, 297, 2030-2033.

(2) Tang, J.; McKellar, A. R. W.; Mezzacapo, F.; Moroni, S. Bridging the Gap between Small Clusters and Nanodroplets: Spectroscopic Study and Computer Simulation of Carbon Dioxide Solvated with Helium Atoms. Phys. Rev. Lett. 2004, 92, 145503.

(3) von Haeften, K.; Laarmann, T.; Wabnitz, H.; Möller, T. The Electronically Excited States of Helium Clusters: an Unusual Example for the Presence of Rydberg States in Condensed Matter. J. Phys. B, At. Mol. Opt. Phys. 2005, 38, S373-S386.

(4) McKellar, A.; Xu, Y.; Jäger, W. Spectroscopic Exploration of Atomic Scale Superfluidity in Doped Helium Nanoclusters. Phys. Rev. Lett. 2006, 97, 183401.

(5) Toennies, J. P.; Vilesov, A. F. Superfluid Helium Droplets: A Uniquely Cold Nanomatrix for Molecules and Molecular Complexes. Angew. Chem. Int. Ed. 2004, 43, 26222648.

(6) Peterka, D. S.; Lindinger, A.; Poisson, L.; Ahmed, M.; Neumark, D. M. Photoelectron Imaging of Helium Droplets. Phys. Rev. Lett. 2003, 91, 043401.

(7) Ziemkiewicz, M. P.; Neumark, D. M.; Gessner, O. Ultrafast Electronic Dynamics in Helium Nanodroplets. Int. Rev. Phys. Chem. 2015, 34, 239-267.

(8) von Haeften, K.; Metzelthin, A.; Rudolph, S.; Staemmler, V.; Havenith, M. HighResolution Spectroscopy of NO in Helium Droplets: A Prototype for Open Shell Molecular Interactions in a Quantum Solvent. Phys. Rev. Lett. 2005, 95, 215301. 
(9) Hartmann, M.; Miller, R. E.; Toennies, J. P.; Vilesov, A. F. Rotationally Resolved Spectroscopy of $\mathrm{SF}_{6}$ in Liquid Helium Clusters: A Molecular Probe of Cluster Temperature. Phys. Rev. Lett. 1995, 75, 1566.

(10) Kwon, Y.; Whaley, K. B. Atomic-Scale Quantum Solvation Structure in Superfluid Helium-4 Clusters. Phys. Rev. Lett. 1999, 83, 4108-4111.

(11) Kwon, Y.; Huang, P.; Patel, M.; Blume, D.; Whaley, K. Quantum Solvation and Molecular Rotations in Superfluid Helium Clusters. J. Chem. Phys. 2000, 113, 6469.

(12) Grebenev, S.; Toennies, J.; Vilesov, A. Superfluidity Within a Small Helium-4 Cluster: The Microscopic Andronikashvili Experiment. Science 1998, 279, 2083.

(13) Surko, C.; Reif, F. Evidence for a New Kind of Energetic Neutral Excitation in Superfluid Helium. Phys. Rev. Lett. 1968, 20, 582.

(14) Dennis, W. S.; E. Durbin, J.; Fitzsimmons, W. A.; Heybey, O.; Walters, G. K. Spectroscopic Identification of Excited Atomic and Molecular States in Electron-Bombarded Liquid Helium. Phys. Rev. Lett. 1969, 23, 1083-1086.

(15) Stockton, M.; Keto, J. W.; Fitzsimmons, W. A. Ultraviolet Emission Spectrum of Electron-Bombarded Superfluid Helium. Phys. Rev. Lett. 1970, 24, 654.

(16) Surko, C. M.; Packard, R. E.; Dick, G. J.; Reif, F. Spectroscopic Study of the Luminescence of Liquid Helium in the Vacuum Ultraviolet. Phys. Rev. Lett. 1970, 24, 657.

(17) Hill, J. C.; Heybey, O.; Walters, G. K. Evidence of Metastable Atomic and Molecular Bubble States in Electron-Bombarded Superfluid Liquid Helium. Phys. Rev. Lett. 1971, 26, 1213-1216. 
(18) Keto, J. W.; Stockton, M.; Fitzsimmons, W. A. Dynamics of Atomic and Molecular Metastable States Produced in Electron-Bombarded Superfluid Helium. Phys. Rev. Lett. 1972, 28, 792-795.

(19) Soley, F.; Fitzsimmons, W. Pressure Shifts and Quenching of Atomic and Molecular States Produced in Electron-Bombarded Liquid Helium. Phys. Rev. Lett. 1974, 32, 988-991.

(20) von Haeften, K.; de Castro, A.; Joppien, M.; Moussavizadeh, L.; von Pietrowski, R.; Möller, T. Discrete Visible Luminescence of Helium Atoms and Molecules Desorbing from Helium Clusters: The Role of Electronic, Vibrational, and Rotational Energy Transfer. Phys. Rev. Lett. 1997, 78, 4371-4374.

(21) von Haeften, K.; Laarmann, T.; Wabnitz, H.; Möller, T. Bubble Formation and Decay in ${ }^{3} \mathrm{He}$ and ${ }^{4} \mathrm{He}$ Clusters. Phys. Rev. Lett. 2002, 88, 233401.

(22) Yurgenson, S.; Hu, C.-C.; Kim, C.; Northby, J. Detachment of Metastable Helium Molecules from Helium Nanodroplets. Eur. Phys. J. D - At., Mol., Opt., Plasma Phys. $1999,9,153-157$.

(23) Mendoza-Luna, L. G.; Watkins, M.; von Haeften, K.; Bonifaci, N.; Aitken, F. Line Broadening of Excimers Bound to the Surface of ${ }^{4} \mathrm{He}$ Clusters Investigated by Comparison with Corona Discharge Excitation Spectra. Eur. J. Phys. D: At., Mol. Clusters 2013, 67, 1-6.

(24) Benderskii, A.; Zadoyan, R.; Schwentner, N.; Apkarian, V. Photodynamics in Superfluid Helium: Femtosecond Laser-Induced Ionization, Charge Recombination, and Preparation of Molecular Rydberg States. J. Chem. Phys. 1999, 110, 1542.

(25) McKinsey, D. N.; Lippincott, W. H.; Nikkel, J. A.; Rellergert, W. G. Trace Detection of Metastable Helium Molecules in Superfluid Helium by Laser-Induced Fluorescence. Phys. Rev. Lett. 2005, 95, 111101. 
(26) Rellergert, W. G.; Cahn, S. B.; Garvan, A.; Hanson, J. C.; Lippincott, W. H.; Nikkel, J. A.; McKinsey, D. N. Detection and Imaging of $\mathrm{He}_{2}$ Molecules in Superfluid Helium. Phys. Rev. Lett. 2008, 100, 025301.

(27) Guo, W.; Wright, J. D.; Cahn, S. B.; Nikkel, J. A.; McKinsey, D. N. Metastable Helium Molecules as Tracers in Superfluid ${ }^{4}$ He. Phys. Rev. Lett. 2009, 102, 235301.

(28) Zmeev, D. E.; Pakpour, F.; Walmsley, P. M.; Golov, A. I.; Guo, W.; McKinsey, D. N.; Ihas, G. G.; McClintock, P. V. E.; Fisher, S. N.; Vinen, W. F. Excimers $\mathrm{He}_{2}^{*}$ as Tracers of Quantum Turbulence in ${ }^{4} \mathrm{He}$ in the $\mathrm{T}=0$ Limit. Phys. Rev. Lett. 2013, 110, 175303.

(29) Guo, W.; La Mantia, M.; Lathrop, D. P.; Van Sciver, S. W. Visualization of Two-Fluid Flows of Superfluid Helium-4. Proc. Nat. Ac. Sci. 2014, 111, 4653-4658.

(30) Gao, J.; Marakov, A.; Guo, W.; Pawlowski, B. T.; Van Sciver, S. W.; Ihas, G. G.; McKinsey, D. N.; Vinen, W. F. Producing and Imaging a Thin Line of $\mathrm{He}_{2}^{*}$ Molecular Tracers in Helium-4. Rev. Sci. Instr. 2015, 86, 093904.

(31) Li, Z.-L.; Bonifaci, N.; Aitken, F.; Denat, A.; von Haeften, K.; Atrazhev, V.; Shakhatov, V. Spectroscopic Investigation of Liquid Helium Excited by a Corona Discharge: Evidence for Bubbles and Red Satellites. Eur. Phys. J. Appl. Phys. 2009, 47.

(32) Ginter, M. L. The Spectrum and Structure of the $\mathrm{He}_{2}$ Molecule: Part III. Characteri-

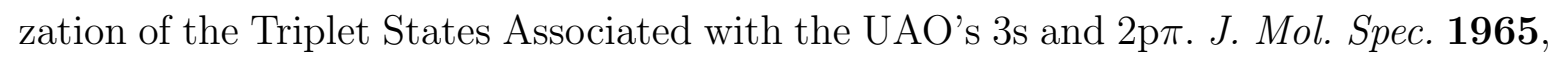
$18,321-343$.

(33) Fontana, P. R. Spin-Orbit and Spin-Spin Interactions in Diatomic Molecules. I. Fine Structure of $\mathrm{H}_{2}$. Phys. Rev. 1962, 125, 220.

(34) Sartakov, B. G.; Toennies, J. P.; Vilesov, A. F. Infrared spectroscopy of Carbonyl Sulfide Inside a Pure ${ }^{3}$ He Droplet. J. Chem. Phys. 2012, 136, 134316. 
(35) Zillich, R. E.; Kwon, Y.; Whaley, K. B. Roton-Rotation Coupling of Acetylene in ${ }^{4}$ He. Phys. Rev. Lett. 2004, 93, 250401-4.

(36) Zillich, R.; Whaley, K. Solvation Structure and Rotational Dynamics of LiH in ${ }^{4} \mathrm{He}$ Clusters. J. Phys. Chem. A 2007, 111, 7489-7498.

(37) von Haeften, K.; Rudolph, S.; Simanovski, I.; Havenith, M.; Zillich, R. E.; Whaley, K. B. Probing Phonon-Rotation Coupling in Helium Nanodroplets: Infrared Spectroscopy of CO and its Isotopomers. Phys. Rev. B 2006, 73, 054502.

(38) Zillich, R. E.; Whaley, K. B.; von Haeften, K. Lineshape of Rotational Spectrum of CO in ${ }^{4}$ He droplets. J. Chem. Phys. 2008, 128, 094303.

(39) Keto, J. W.; Soley, F. J.; Stockton, M.; Fitzsimmons, W. A. Dynamic Properties of Neutral Excitations Produced in Electron-Bombarded Superfluid Helium. II. Afterglow Fluorescence of Excited Helium Molecules. Phys. Rev. A 1974, 10, 887.

(40) Kiryukhin, V.; Keimer, B.; Boltnev, R. E.; Khmelenko, V. V.; Gordon, E. B. Inert-Gas Solids with Nanoscale Porosity. Phys. Rev. Lett. 1997, 79, 1774.

(41) Lebedev, V.; Moroshkin, P.; Toennies, J.; Weis, A. Spectroscopy of the Copper Dimer in Normal Fluid, Superfluid, and Solid He. J. Chem. Phys. 2010, 133, 154508.

(42) Khmelenko, V. V.; Mao, S.; Meraki, A.; Wilde, S. C.; McColgan, P.; Pelmenev, A. A.; Boltnev, R. E.; Lee, D. M. Luminescence of Oxygen Atoms Stimulated by Metastable Helium at Cryogenic Temperatures. Phys. Rev. Lett. 2013, 111, 183002.

(43) Popov, E.; Eloranta, J. Copper Dimer Interactions on a Thermomechanical Superfluid ${ }^{4}$ He Fountain. J. Chem. Phys. 2015, 142, 204704.

(44) Katsuki, H.; Momose, T. Observation of Rovibrational Dephasing of Molecules in Parahydrogen Crystals by Frequency Domain Spectroscopy. Phys. Rev. Lett. 2000, 84, 3286-3289. 
(45) Katsuki, H.; Fushitani, M.; Momose, T. Quantum Property of Solid Hydrogen as Revealed by High-Resolution Laser Spectroscopy. Low. Temp. Phys. 2003, 29, 832-837.

(46) Lorenz, B. D.; Anderson, D. T. Infrared Spectra of $\mathrm{N}_{2} \mathrm{O}-\left(\text { ortho- } \mathrm{D}_{2}\right)_{N}$ and $\mathrm{N}_{2} \mathrm{O}-(\mathrm{HD})_{N}$ Clusters Trapped in Bulk Solid Parahydrogen. J. Phys. Chem. 2007, 126, 184506184506.

(47) Mendoza Luna, L. G. Free and Hindered-Rotation of Helium Excimers in Liquid Helium via a Bulk Experiment. Ph.D. Thesis, University of Leicester, Leicester, UK, 2015. 